\title{
Space and Time Shape Constrained Deformable Surfaces for 4D Medical Image Segmentation
}

\author{
Johan Montagnat and Hervé Delingette \\ INRIA Sophia, BP 93, 06902 Sophia-Antipolis Cedex, France \\ http://www-sop.inria.fr/epidaure/
}

\begin{abstract}
The aim of this work is to automatically extract quantitative parameters from time sequences of $3 \mathrm{D}$ images (4D images) suited to heart pathology diagnosis. In this paper, we propose a framework for the reconstruction of the left ventricle motion from 4D images based on $4 \mathrm{D}$ deformable surface models. These 4D models are represented as a time sequence of 3D meshes whose deformation are correlated during the cardiac cycle. Both temporal and spatial constraints based on prior knowledge of heart shape and motion are combined to improve the segmentation accuracy. In contrast to many earlier approaches, our framework includes the notion of trajectory constraint. We have demonstrated the ability of this segmentation tool to deal with noisy or low contrast images on 4D MR, SPECT, and US images.
\end{abstract}

\section{Context}

Recently, the improvement of medical image acquisition technology has allowed the production of time sequences of 3D medical images (4D images) for several image modalities (CT, MRI, US, SPECT). Tagged MRI is the gold standard of heart motion analysis since it is the only modality permitting the extraction of the motion of physical points located in the myocardium [18]. However, other modalities may be used for meaningful parameters extraction at a lower cost. In particular, the fast development of 3D US imaging is very promising due to its accessibility and low cost [17].

The main target for these new ultra-fast image acquisition devices is to capture and analyze the heart motion through the extraction of quantitative parameters such as volume, walls thickness, ejection fraction and motion amplitude. In order to estimate these parameters, it is necessary to reconstruct the Left Ventricle (LV) motion during a cardiac cycle. Tracking the LV motion in 2D or $3 \mathrm{D}$ image sequences has led to several research efforts $[10,9,2]$. Tracking $[12,16]$ and motion analysis $[4,7]$ based on deformable models in $4 \mathrm{D}$ images take into account time continuity and periodicity to improve their robustness.

In this paper, we propose to track the LV based on 4D deformable models. Our concept of 4D deformable surface models combines spatial and temporal constraints which differs from most previous approaches $[12,16,4]$ that decouple them. Furthermore, in contrast to the strategy presented in [7], the motion estimation is not parameterized by a global time-space transformation. It leads to 
more efficient computation and to greater descriptive ability in motion recovery. Finally, our approach can include the notion of trajectory constraint which is the generalization of the shape constraint.

\section{4D Deformable Models}

Let $I$ denote a 4D image composed of $n$ volume images corresponding to $n$ different time points $\left\{t_{0}, \ldots, t_{n-1}\right\}$. We define a $4 \mathrm{D}$ deformable model $\mathcal{S}$ as a set of $n$ deformable surfaces $\left\{\mathcal{S}_{t}\right\}_{t \in[0, n-1]}$, each surface model $\mathcal{S}_{t}$ representing a given anatomical structure at time point $t$. Among the possible geometric representations of deformable surfaces [13], we have chosen the simplex meshes [8] discrete surfaces. They are defined by a set of vertices and a constant connectivity function. Their main advantage lies in their simple data structure permitting an efficient implementation both in terms of computational time and memory storage. This is specifically important in the case of $4 \mathrm{D}$ deformable models where $n$ surface meshes must be updated at each iteration. Furthermore, simplex meshes are especially well-suited for the computation of curvature-based regularizing forces. All $n$ surface meshes $\mathcal{S}_{t}$ have the same topology, i.e. there is a one to one correspondence between the $d$ vertices composing each surface. In the rest of the paper, $\mathbf{p}_{i, t}$ denotes the position of vertex number $i$ at time $t$. While the model undergoes deformations, each surface $\mathcal{S}_{t}$ evolves in space but it remains at its time step (i.e., $t$ does not change).

A 4D model deforms under the combined action of three forces aiming at recovering the shape and motion of an anatomical structure: (i) the data, or external, force attracts each vertex towards the structure boundaries; (ii) the spatial regularizing, or internal, force ensures the smoothness of the deforming surface by introducing spatial continuity constraints in the deformation process; (iii) the temporal regularizing force similarly relies on prior knowledge on the time dimension continuity to regularize the deformation process. A second order (Newtonian) evolution scheme discretized using an explicit scheme governs the displacement of each vertex (see [8] for details):

$\mathbf{p}_{i, t}^{k+\Delta k}=\mathbf{p}_{i, t}^{k}+(1-\gamma)\left(\mathbf{p}_{i, t}^{k}-\mathbf{p}_{i, t}^{k-\Delta k}\right)+\alpha_{i} f_{\text {int }}\left(\mathbf{p}_{i, t}^{k}\right)+\delta_{i} f_{\text {time }}\left(\mathbf{p}_{i, t}^{k}\right)+\beta_{i} f_{\text {ext }}\left(\mathbf{p}_{i, t}^{k}\right)$,

where $f_{\text {int }}, f_{\text {time }}$, and $f_{\text {ext }}$ are the internal, the temporal, and external forces respectively. $\alpha_{i}, \beta_{i}$, and $\delta_{i}$ are weights including the vertex mass and the iteration step $\Delta k$. In all our experiments, the background damping $\gamma$ is fixed to value 0.35 based on an empirical study showing that this value optimizes the convergence speed in general. The $\alpha_{i}$ values are always fixed to 1 .

Simplex meshes provide a powerful framework for computing internal regularizing forces [8] including smoothing forces without shrinking side effect. External forces are computed as distance functions of the model vertices to the data. This speeds-up the model convergence compared to potential fields approaches and it avoids oscillations [5]. Deformations are computed along each vertex normal direction to avoid creating distorted meshes. Both gradient based and region based criteria are used to determine boundary voxels in images. For the sake 
of brevity, the external forces computation is not discussed here and the reader may refer to [15] for details.

\section{Shape and Temporal Constraints}

The main incentive for performing medical image segmentation based on deformable models lies in their ability to incorporate prior knowledge on the data that is being recovered. In most cases, this knowledge is translated mathematically into a set of regularizing constraints that greatly improves the robustness and accuracy of the segmentation process. Indeed, many methods have been proposed to regularize deformations by limiting the model number of parameters $[20,6]$, or controlling the kind of deformation applied onto the model $[3,11]$.

We introduce two complementary constraints that are specifically suited for the $\mathrm{LV}$ tracking in $4 \mathrm{D}$ images. The former consists of a shape constraint that tends to enforce 3D geometric continuity. The latter is a temporal constraint that causes a 4D mesh to rely on prior motion knowledge. It is important to note that, in contrast to many previous works, both constraints are applied simultaneously thus leading to a true 4D approach. Furthermore, each constraint can encapsulate a weak or strong prior knowledge, as summarized in table 1.

\begin{tabular}{|c|c|c|}
\hline Prior knowledge & Spatial constraint & Temporal constraint \\
\hline Weak & Curvature-based shape smoothing & Temporal position averaging \\
\hline Strong & Shape constraint & Trajectory constraint \\
\hline
\end{tabular}

Table 1. Spatial and temporal constraint depending of the amount of prior knowledge.

\subsection{Shape Constraints}

In the case where no reference shape is known (weak shape constraint), we use the regularizing force defined in [8], that minimizes the variation of mean-curvature over the mesh. Otherwise, we add an additional shape constraint force $f_{\text {shape }}$ that is related to a reference shape $\mathcal{S}^{\prime}$ of the anatomical structure. It introduces shape prior knowledge by extending the globally-constrained deformation scheme described in [14] to the 4D case. Let $\mathcal{S}^{k}$ denotes the 4D model after the $k^{\text {th }}$ iteration. At initialization, $\mathcal{S}^{\prime 0}=\mathcal{S}^{0}$. At each iteration, external forces $f_{\text {ext }}$ are computed for each vertex so that $\mathbf{p}_{i, t}+f_{\text {ext }}\left(\mathbf{p}_{i, t}\right)$ corresponds to the myocardium boundary point that best matches $\mathbf{p}_{i, t}$. We estimate a global transformation $T^{k}$ belonging to a given group of transformations (e.g. affine transformations $\mathrm{T}_{\text {affine }}$ ). $T^{k}$ approximates the external force field by minimizing the least square criterion:

$$
T^{k}=\arg \min _{T \in \mathrm{T}_{\text {affine }}}\left\{\sum_{t=0}^{n-1} \sum_{i=0}^{d-1}\left\|T\left(\mathbf{p}_{i, t}\right)-\left(\mathbf{p}_{i, t}+f_{\text {ext }}\left(\mathbf{p}_{i, t}\right)\right)\right\|^{2}\right\} .
$$

We then update the reference shape: $\mathcal{S}^{\prime k+\Delta k}=T^{k} \circ T^{k-1} \ldots \circ T^{1}\left(\mathcal{S}^{\prime 0}\right)$. Thus $\mathcal{S}^{\prime k+\Delta k}$ remains identical to $\mathcal{S}^{\prime 0}$ up to an affine transformation. A shape force is 
defined on each vertex of $\mathcal{S}^{k}$ as a spring-like force towards its updated reference position: $f_{\text {shape }}\left(\mathbf{p}_{i, t}^{k}\right)=\mathbf{p}_{i, t}^{k} \mathbf{p}_{i, t}^{\prime k}$.

Furthermore, a locality parameter $\lambda$ is introduced to weight the influence of the shape force relative to the internal and external forces as described in [14]:

$$
\begin{aligned}
\mathbf{p}_{i, t}^{k+\Delta k}= & \mathbf{p}_{i, t}^{k}+(1-\gamma)\left(\mathbf{p}_{i, t}^{k}-\mathbf{p}_{i, t}^{k-\Delta k}\right)+ \\
& \lambda\left(\alpha_{i} f_{\text {int }}\left(\mathbf{p}_{i, t}^{k}\right)+\delta_{i} f_{\text {time }}\left(\mathbf{p}_{i, t}^{k}\right)+\beta_{i} f_{\text {ext }}\left(\mathbf{p}_{i, t}^{k}\right)\right)+(1-\lambda) f_{\text {shape }}\left(\mathbf{p}_{i, t}^{k}\right) .
\end{aligned}
$$

When $\lambda=0$, the $4 \mathrm{D}$ model is deformed through the application of a global transformation from its reference shape, thus making the deformation process robust to noise and outliers. Conversely, if $\lambda=1$, only the weak shape constraint applies and the model shape variability is very high. Any intermediate value of $\lambda$ produces local deformations combined with a global shape constraint.

\subsection{Temporal Constraints}

The temporal regularizing force $f_{\text {time }}$ is defined as a spring-like force $f_{\text {time }}\left(\mathbf{p}_{i, t}\right)=$ $\tilde{\mathbf{p}}_{i, t}-\mathbf{p}_{i, t}$ attracting vertex $\mathbf{p}_{i, t}$ towards a reference point $\tilde{\mathbf{p}}_{i, t}$. When no prior knowledge is used, we define a weak temporal constraint by attracting $\mathbf{p}_{i, t}$ towards the middle position of its two temporal neighbors: $\tilde{\mathbf{p}}_{i, t}=\frac{\mathbf{p}_{i, t-1}+\mathbf{p}_{i, t+1}}{2}$. Applying this force is equivalent to minimizing the speed of each vertex, and therefore to minimizing the kinetic energy of the 4D model.

When using prior information on the trajectory of each vertex, we determine $\tilde{\mathbf{p}}_{i, t}$ such that this point lies on the ideal vertex trajectory. It is important to note that these trajectories usually do not correspond to trajectories of physical points lying on the myocardium but are only used as mathematical constraints. To store prior trajectories, we could store the $n$ vertex positions $\left\{\mathbf{p}_{i, t}\right\}_{t \in[0, n-1]}$ of each vertex over time. However, this representation would imply that the trajectory orientation and scale is constant between images, which is not the case. Instead, we choose to store the 3D curve trajectory as a set of geometric parameters $\left\{\varepsilon_{i, t}, \varphi_{i, t}, \psi_{i, t}\right\}$ that are invariant to rotation, translation, and scale. The left side of Fig. 1 illustrates the elements composing the trajectory geometry.

Let $\mathbf{p}_{i, t}^{\perp}$ denote the orthogonal projection of $\mathbf{p}_{i, t}$ onto its two temporal neighbors segment $\left[\mathbf{p}_{i, t-1}, \mathbf{p}_{i, t+1}\right]$. The position of point $\mathbf{p}_{i, t}$ may be defined through: (i) the metric parameter $\varepsilon_{i, t}$ measuring the relative position of $\mathbf{p}_{i, t}^{\perp}$ in segment $\left[\mathbf{p}_{i, t-1}, \mathbf{p}_{i, t+1}\right]$; (ii) the angle $\varphi_{i, t}$ measuring the elevation of $\mathbf{p}_{i, t}$ above the segment $\left[\mathbf{p}_{i, t-1}, \mathbf{p}_{i, t+1}\right]$ in the plane $\left(\mathbf{p}_{i, t-1}, \mathbf{p}_{i, t}, \mathbf{p}_{i, t+1}\right)$; and (iii) the angle $\psi_{i, t}$ measuring the discrete torsion. Intuitively, $\varepsilon_{i, t}, \varphi_{i, t}$, and $\psi_{i, t}$ correspond to discrete arc length, curvature, and torsion respectively. Let $\mathbf{t}_{i, t}$ denote the discrete tangent, $\mathbf{b}_{i, t}$ the binormal vector, and $\mathbf{n}_{i, t}$ the discrete normal to point $\mathbf{p}_{i, t}$ respectively:

$$
\mathbf{t}_{i, t}=\frac{\mathbf{p}_{i, t-1} \mathbf{p}_{i, t+1}}{\left\|\mathbf{p}_{i, t-1} \mathbf{p}_{i, t+1}\right\|}, \mathbf{b}_{i, t}=\frac{\mathbf{p}_{i, t} \mathbf{p}_{i, t+1} \wedge \mathbf{p}_{i, t-1} \mathbf{p}_{i, t}}{\left\|\mathbf{p}_{i, t} \mathbf{p}_{i, t+1} \wedge \mathbf{p}_{i, t-1} \mathbf{p}_{i, t}\right\|}, \quad \mathbf{n}_{i, t}=\mathbf{b}_{i, t} \wedge \mathbf{t}_{i, t} .
$$



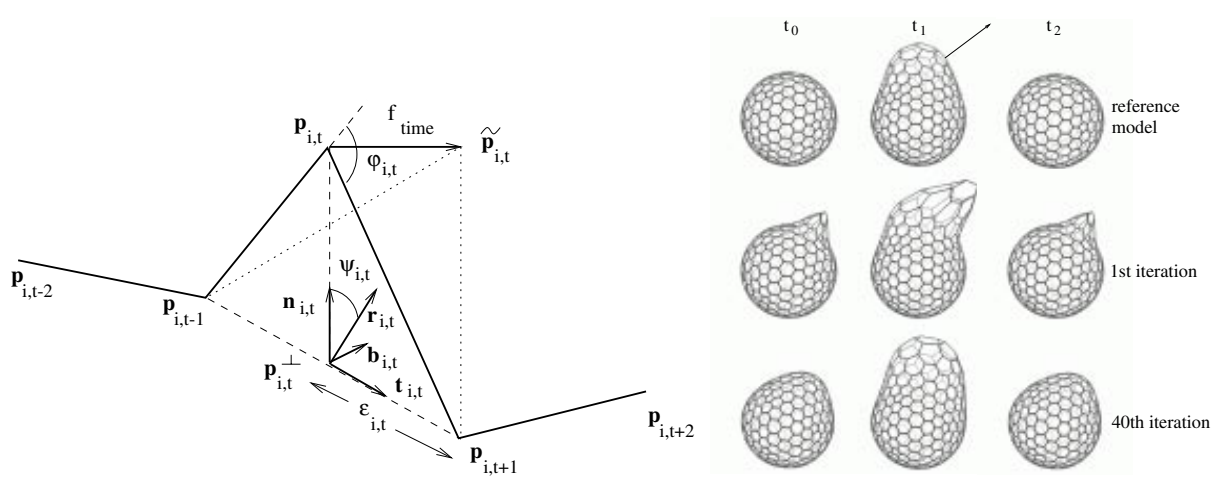

Fig. 1. Left: trajectory geometry and temporal force. Right: temporal force effect.

The metric parameter is defined by $\varepsilon_{i, t}=\frac{\left\|\mathbf{p}_{i, t}^{\perp} \mathbf{p}_{i, t+1}\right\|}{\left\|\mathbf{p}_{i, t-1} \mathbf{p}_{i, t+1}\right\|}$, the elevation angle is $\varphi_{i, t}=\left(\mathbf{p}_{i, t} \mathbf{p}_{i, t+1}, \mathbf{p}_{i, t-1} \mathbf{p}_{i, t}\right)$, and the torsion angle is such that:

$\mathbf{r}_{i, t}=\frac{\mathbf{t}_{i, t} \wedge\left(\mathbf{p}_{i, t-2} \mathbf{p}_{i, t-1} \wedge \mathbf{p}_{i, t+1} \mathbf{p}_{i, t+2}\right)}{\left\|\mathbf{t}_{i, t} \wedge\left(\mathbf{p}_{i, t-2} \mathbf{p}_{i, t-1} \wedge \mathbf{p}_{i, t+1} \mathbf{p}_{i, t+2}\right)\right\|}$ and $\mathbf{n}_{i}=\cos \left(\psi_{i, t}\right) \mathbf{r}_{i, t}+\sin \left(\psi_{i, t}\right) \mathbf{t}_{i, t} \wedge \mathbf{r}_{i, t}$.

The position of $\mathbf{p}_{i, t}$ is related to the position of its neighbors and the trajectory parameters by equation:

$$
\begin{aligned}
\mathbf{p}_{i, t}= & \varepsilon_{i, t} \mathbf{p}_{i, t-1}+\left(1-\varepsilon_{i, t}\right) \mathbf{p}_{i, t+1}+ \\
& h\left(\varepsilon_{i, t}, \varphi_{i, t}, \mathbf{p}_{i, t-1}, \mathbf{p}_{i, t+1}\right)\left(\cos \left(\psi_{i, t}\right) \mathbf{r}_{i, t}+\sin \left(\psi_{i, t}\right) \mathbf{t}_{i, t} \wedge \mathbf{r}_{i, t}\right),
\end{aligned}
$$

where $h=\left\|\mathbf{p}_{i, t} \mathbf{p}_{i, t}^{\perp}\right\|$. The temporal force is computed with $\tilde{\mathbf{p}}_{i, t}$ defined by equation 3 using the trajectory reference parameters.

The right side of Fig. 1 shows the temporal constraint effect. A spherical 4D model composed of 3 time points $\left(t_{0}, t_{1}\right.$, and $\left.t_{2}\right)$ is shown in the upper row. A single vertex of the model is submitted to an external force at time $t_{1}$. The middle row shows the resulting deformation. Surface $\mathcal{S}_{1}$ is deformed causing surfaces $\mathcal{S}_{0}$ and $\mathcal{S}_{2}$ to deform through the temporal constraint, although the deformation is attenuated in time. The bottom row shows the surface converging towards its reference motion after 40 iterations.

\subsection{Initialization Procedure}

In general, to get a first rough position of the $4 \mathrm{D} \mathrm{LV}$ model, we first proceed by using only highly constrained spatial deformations without any temporal constraint. By using $\lambda=0$, we basically estimate a set of global affine transformations to align the model with the 4D dataset. Then, we proceed by iteratively increasing the locality parameter $\lambda$ while adding temporal constraints. This approach allows an evolutional deformation scheme based on a coarse-tofine strategy. 


\section{4D Medical Image Segmentation}

The 4D model described above has been used to segment 4D MR, SPECT and US images. Figure 2 shows two slices of each image modality at the end of diastole and the end of systole. Due to real time imaging constraints, all images have a sparse resolution. Cardiac MR images have a very high resolution in slice planes. However, the third dimension resolution is much lower $(256 \times 256 \times 9)$. SPECT images are sampled on a $64^{3}$ voxel grid. Finally, the 4D US images shown are composed by a set of slices acquired with a rotative probe [15] leading to a low spatial resolution $(256 \times 256 \times 9$, with a 20 degrees angle between two slices $)$.

The $4 \mathrm{D}$ model used are made of 500 to 700 vertices per surface. This rough resolution is adapted to the images level of detail. In MR and US images, the internal wall of the LV is reconstructed by a closed surface representing the internal blood volume. In the case of SPECT images, the internal and external walls of the LV appear. A cup shaped surface model is then used. The US image sequences are composed of 8 time points covering only the systole while the MR (13 time points) and the SPECT ( 8 time points) sequences cover a complete heart cycle.
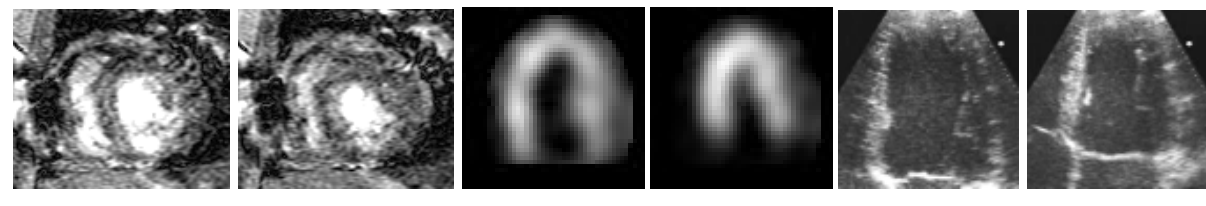

Fig. 2. MR (left), SPECT (centre), and US (right) slices at end diastole and end systole

\subsection{MR Images}

We show a segmentation experiment on one heart beat sequence. The cardiac MR images contrast varies between slices and the heart boundaries are poorly defined. A 4D model is generated by embedding a set of identical ellipsoids roughly centered on the LV in the first image sequence. Only spatial and temporal smoothing (weak) constraints are used since no relevant prior information was given. The local deformations are constrained by a global affine transformation. The coarse-to-fine deformation algorithm involves two stages composed of 30 iterations each: $\lambda=10 \%$ and $\beta=0.5$ followed by $\lambda=40 \%$ and $\beta=0.1$. The weight values $\alpha=1$ and $\delta=0.1$ are fixed. The low $\lambda$ value prevents the surface from being too sensitive to the lack of information in area where the gradient filter gave no response.

Each surface model is composed of 500 vertices and the deformation process for the whole $4 \mathrm{D}$ model only takes $1 \mathrm{~min} 46 \mathrm{~s}$ on a $500 \mathrm{MHz}$ Digital PWS with $512 \mathrm{Mb}$ of memory. Four out of the 13 surfaces composing the 4D model are shown in Fig. 3 on the left. The middle graph plots the curve of volume variation through time. It corresponds to an healthy case volume curve. 

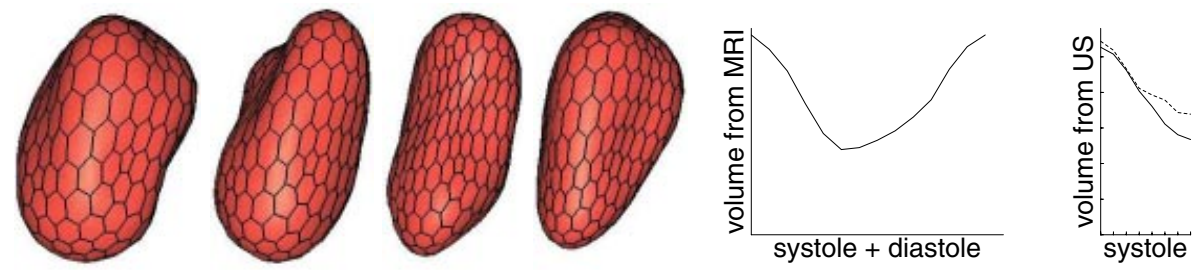

Fig. 3. Left: 4 surfaces from the 4D models deformed in an MRI. Middle: 4D model volume curve. Right: 4D model volume obtained from a US image by 3D (dashed line) and 4D (solid line) segmentation.

\subsection{SPECT Images}

We have processed a 4D SPECT image database including healthy and pathological patients. The systole is approximatively three time points long whereas the diastole takes the remaining five time points. We compare images of healthy patients with a normal endocardium blood perfusion and pathological patients with an abnormal perfusion due to some ischemic zones. The mean deformation time for all 4D models, made of 700 vertex surfaces, is 2 min $34 \mathrm{~s}$.

The reference model is built from an healthy patient image by $3 \mathrm{D}$ segmentation. The high image contrast allows us to use gradient information to compute external forces. The 3D segmentation does not involve any time continuity constraints. A 4D deformation stage with time smoothing forces is therefore needed to obtain a reference model with reliable shape and motion. Shape constraints are especially beneficial for the segmentation of low contrasted images showing pathologies such as ischemia. Due to the similarity between images, the 4D model is roughly initialized in its reference position. Rigid then similarity registration are first used to compensate the differences in location and size between patients $(\alpha=1, \beta=0.1, \delta=0.2)$. Local deformations with an affine constraint are then used. The deformation involves 3 stages (20 iterations each) in the coarse-to-fine algorithm: the locality increases $(\lambda=20 \%, 50 \%, 70 \%)$ while the external force range decreases (range $=4,2,2$ voxels).

Figure 4 shows a frontal view of the 4D models. Top line displays the reference model obtained by 3D segmentation and revealing poor time continuity. The center line displays the 4D model regularized by time smoothing constraints in the image of a healthy patient. The bottom line shows the model extracted from a pathological case by $4 \mathrm{D}$ segmentation. The surface model reveals the pathological heart with weak motion amplitude.

\subsection{Ultrasound Images}

The speckle noise of US images and the lack of beam reflection on boundaries tangent to the US rays make the segmentation process difficult. A $4 \mathrm{D}$ model is built by $3 \mathrm{D}$ segmentation of a 8 time points reference image. The model is 


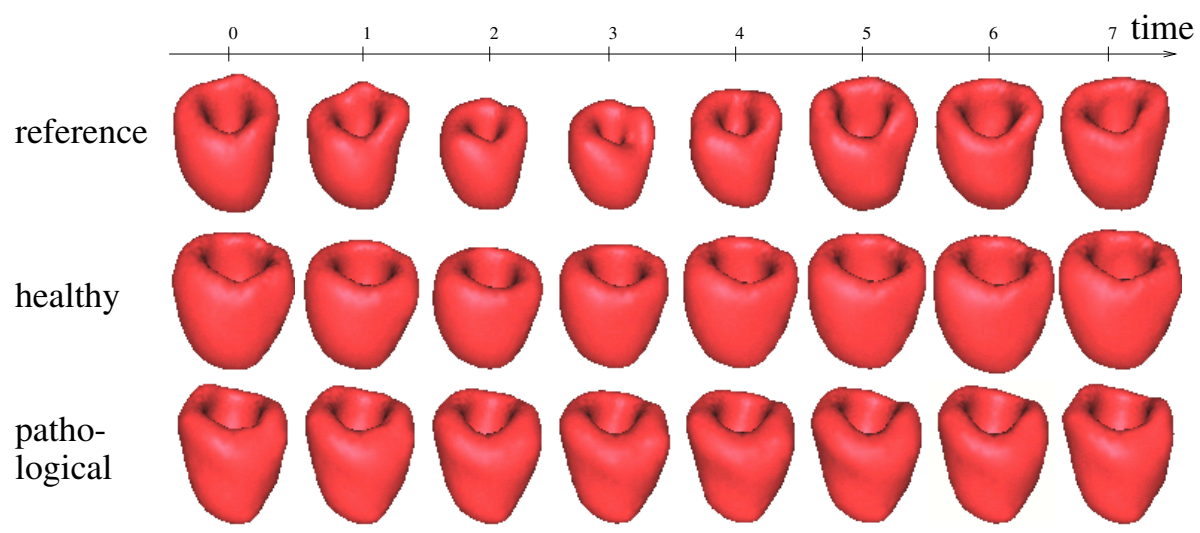

Fig. 4. 4D models of the myocardium: reference model obtained by 3D segmentation (top row), healthy case (middle row), and pathological case (bottom row).

first registered by a similarity transformation to align and adapt its scale to the data. The gradient information is sufficient since the deformations are strongly constrained to speed-up the model convergence. A large force range allows the model to find boundaries far from the initial model position. After registration, the model locally deforms with an affine global constraint $(\alpha=1, \beta=0.5$, $\delta=0.1$, range $=20$ voxels). Local deformations guided by region based forces are used in the final segmentation. Region-based forces slow down the deformation process and the total reconstruction time was 4 min $42 \mathrm{~s}$.

Right of Fig. 3 shows the evolution of the LV volume through time (solid line). The volume is compared to the result of an iterative $3 \mathrm{D}$ segmentation of the same sequence from an earlier study [15] (dashed line). The time regularizing constraints make the $4 \mathrm{D}$ curve much more regular. The initial volume value is very close ( $3 \%$ difference) but it grows with time. This is not surprising since the $3 \mathrm{D}$ segmentation tends to accumulate errors. Moreover, the $4 \mathrm{D}$ curves shows a profile closer from the theoretical line expected. The model volume leads to a $49 \%$ ejection fraction. This value compares to the $45 \%$ ejection fraction computed from a manual segmentation by a cardiologist on the same sequence.

Figure 5 shows the sequence slices on which are superimposed the model intersections with each plane. The 8 figure columns correspond to the 8 time points. Five rows corresponding to one slice out of two (from top to bottom: 0, $40,80,120$, and 160 degrees of arc) are shown.

\section{Conclusion}

We have demonstrated the ability of $4 \mathrm{D}$ models to track the $\mathrm{LV}$ motion in $4 \mathrm{D}$ noisy medical images. The proposed framework relies on complementary spatial and temporal constraints to regularize the deformation while introducing 
prior knowledge about the LV shape and motion in the segmentation process. Shape constraints allow the segmentation of sparse and low contrast data. The deformable models approach is generic and allows us to deal with different image modalities. In all examples shown above, the algorithm leads to a fully automatic segmentation of the LV once the weighting parameters have been fixed for each image modality.

The resulting surface models are well suited for estimating quantitative parameters such as endocardium volume or wall thickness. Visual results and quantitative measures extracted are reasonable although a thorough clinical study would be necessary to validate the algorithm accuracy. Comparison to earlier work involving 3D segmentation [15] demonstrates the interest of a full 4D approach.

\section{Acknowledgements}

We are grateful to GE Medical Systems, Prof. Goris of the University of Stanford Medical School and Dr Lethor of CHU Barbois for MR, SPECT and US image acquisitions respectively. Special thanks go to Michel Audette and Tal Arbel for proof-reading.
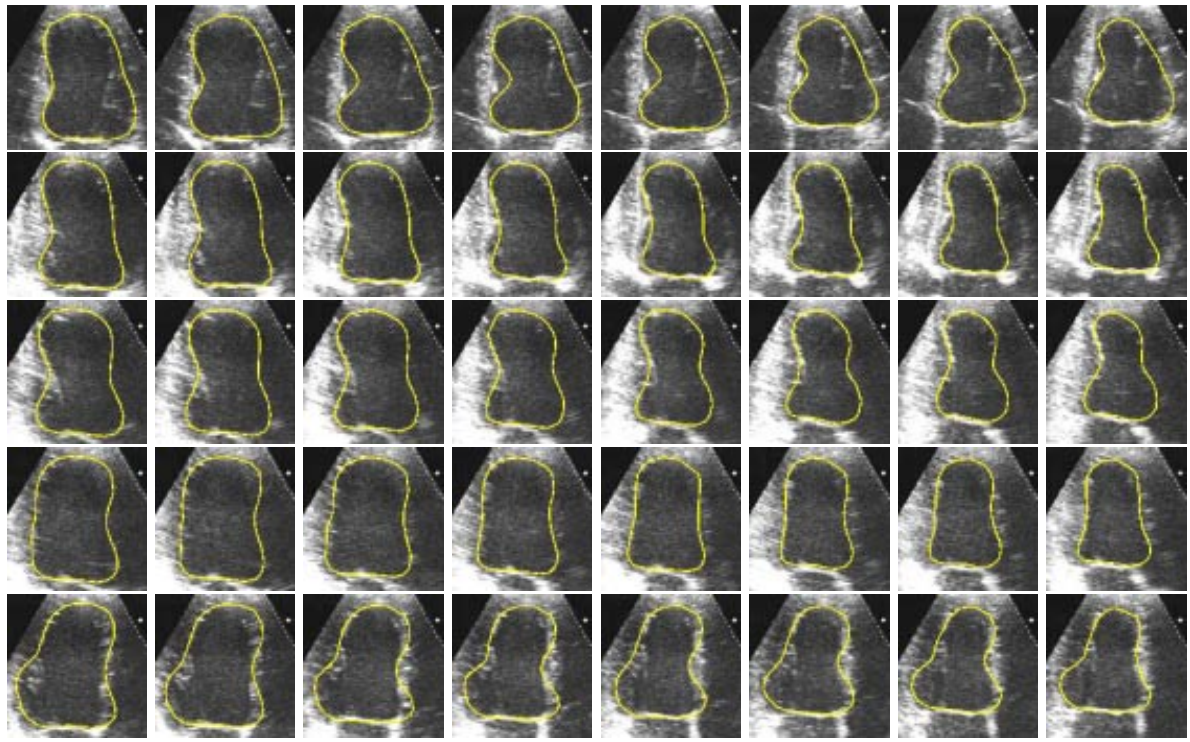

Fig. 5. Set of slices of the US image superimposed with the deformed model intersection. From left to right: cardiac sequence time point. From top to bottom: slices oriented with angle $0,40,80,120$, and 160 degrees of arc. 


\section{References}

1. Bardinet, E., Cohen, L., Ayache, N.: Tracking and motion analysis of the left ventricle with deformable superquadrics. Med. Image Analysis, 1(2) (1996) 129-149

2. Berger, M.-O., Winterfeldt, G., Lethor, J.-P.: Contour Tracking in Echocardiographic Sequences without Learning Stage: Application to the 3D Reconstruction of the 3D Beating Left Ventricle. In MICCAI, vol. 1679 of LNCS, (1999) 508-515

3. Besl, P., McKay, N.: A method for registration of 3D shapes. IEEE Transaction on Pattern Analysis and Machine Intelligence, 14(2) (1992) 239-256

4. Clarysse, P., Friboulet, D., and Magnin, I.: Tracking Geometrical Descriptors on 3-D Deformable Surfaces: Application to the Left-Ventricular Surface of the Heart. IEEE Transaction on Medical Imaging, 16(4) (1997) 392-404

5. Cohen, L.: On Active Contour Models and Balloons. Computer Vision, Graphics, and Image Processing: Image Understanding, 53(2) (1991) 211-218

6. Cootes, T., Taylor, C., Cooper, D., Graham, J.: Active shape models, their training and application. Comp. Vision and Image Understanding, 61(1) (1995) 38-59

7. Declerck, J., Feldmar, J., Ayache, N.: Definition of a 4D continuous planispheric transformation for the tracking and the analysis of LV motion. Medical Image Analysis, 2(2) (1998) 197-213

8. Delingette, H.: General Object Reconstruction based on Simplex Meshes. International Journal of Computer Vision, 32(2) (1999) 111-146

9. Giachetti, A.: On-line analysis of echocardiographic image sequences. Medical Image Analysis, 2(3) (1998) 261-284

10. Jacob, G., Noble, A., Mulet-Parada, M., and Blake, A.: Evaluating a robust contour tracker on echocardiographic sequences. Medical Image Analysis, 3(1) (1999) 63-75

11. Lötjönen, J., Reissman, P.-J., Magnin, I., Katila, T.: Model extraction from magnetic resonance volume data using the deformable pyramid. Medical Image Analysis, 3(4) (1999) 387-406

12. McEachen, J., Duncan, J.: Shaped-base tracking of left ventricular wall motion. IEEE Transaction on Medical Imaging, 16(3) (1997) 270-283

13. McInerney, T., Terzopoulos, D.: Deformable models in medical image analysis: a survey. Medical Image Analysis, 1(2) (1996) 91-108

14. Montagnat, J., Delingette, H.: Globally constrained deformable models for 3D object reconstruction. Signal Processing, 71(2) (1998) 173-186

15. Montagnat, J., Delingette, H., Malandain, G.: Cylindrical Echocardiographic Images Segmentation based on 3D Deformable Models. In MICCAI, vol. 1679 of LNCS, (1999) 168-175

16. Nastar, C., Ayache, N.: Frequency-Based Nonrigid Motion Analysis: Application to Four Dimensional Medical Images. IEEE Transaction on Pattern Analysis and Machine Intelligence, 18(11) (1996) 1067-1079

17. Papademetris, X., Sinusas, A., Dione, D., Duncan, J.: 3D Cardiac Deformation from Ultrasound Images. In MICCAI, vol. 1679 of LNCS, (1999) 420-429

18. Park, J., Metaxas, D., Axel, L.: Analysis of LV motion based on volumetric deformable models and MRI-SPAMM. Medical Image Analysis, 1(1) (1996) 53-71

19. Terzopoulos, D., Witkin, A., Kass, M.: Constraints on Deformable Models: Recovering 3D Shape and Nonrigid Motion. Artificial Intelligence, 36(1) (1988) 91-123

20. Vemuri, B.C., Radisavljevic, A.: From Global to Local, a Continuum of Shape Models with Fractal. In Computer Vision and Pattern Recognition, (1993) 307-313 